\title{
PHYSICAL REVIEW A
}

\author{
GENERAL PHYSICS
}

\section{Nonlinear Schrödinger equation and dissipative quantum dynamics in periodic fields}

\author{
Youhong Huang* and Shih-I Chu \\ Department of Chemistry, University of Kansas, Lawrence, Kansas 66045 \\ Joseph O. Hirschfelder \\ Department of Chemistry, University of Wisconsin, Madison, Wisconsin 53706 \\ (Received 2 February 1989; revised manuscript received 1 May 1989)
}

\begin{abstract}
The nonlinear dynamics of dissipative quantum systems in periodic fields is studied in the framework of a Gisin-like nonlinear Schrödinger equation with deterministic nonunitary quantum friction terms describing the system-bath couplings. The virtue of this nonunitary evolution is that it is compatible with Dirac's superposition principle and the Hilbert-space structure of quantum kinematics. Floquet theory and the generalized Van Vleck nearly degenerate perturbation method are used to facilitate both analytical and numerical solutions. Closed-form analytic solutions can be obtained in the long-time average approximation or within the rotating-wave approximation. The methods are applied to the study of dissipative quantum dynamics of two-level systems driven by intense periodic fields. It is found that the system asymptotically approaches a limit cycle (whose orientation is subject to the quantum friction constraint), regardless of the strength of the perturbed fields and the nonlinearity constant, indicating quantum suppression of classical chaos. Further, each point of the limit cycle is found to be an attractor and $\psi(t)$ exhibits a fractal-like evolution pattern in the course of time. The structure of the limit cycle depends strongly upon field intensity and frequency as well as the order of nonlinear multiphoton transitions. The power spectrum of the Bloch vector trajectory exhibits a dynamical symmetry inherent in the dissipative system and in the asymptotic limit cycle. A theoretical analysis is presented for the understanding of the origin and the role of the dynamical symmetry.
\end{abstract}

\section{INTRODUCTION}

Nonlinear Schrödinger equations with the generic form

$$
\left[\hat{H}_{0}+\hat{V}(\psi)\right] \psi=E \psi
$$

have been used to describe quantum-mechanical systems that interact with their surroundings. ${ }^{1-3}$ Through its wave function $\psi$, the system interacts with its environment by inducing a net field which then acts back on the system itself. This "self-dependent" interaction can be described by appropriate choice of the effective potential $V(\psi)$. In general, one can assume $\hat{V}(\psi)$ depends on the wave function $\psi$ through the expectation value of an operator. This assumption leads to ${ }^{4}$

$$
\left(\hat{H}_{0}+k\langle\psi|\hat{A}| \psi\rangle \hat{B}\right) \psi=E(k) \psi,
$$

where $\hat{A}$ and $\hat{B}$ are operators depending upon the physical model and $k$ is a parameter characterizing the system-bath interaction strength. One physical example leading to such an equation is the problem of a molecule in a polarizable medium, treated by the classical Kirk- wood and Onsager interaction model. ${ }^{5}$ In this case, $\widehat{A}=\widehat{B}=\widehat{M}$, the dipole-moment operator of the system. The parameter $k$ could describe the solute-solvent interaction which depends upon the mean dielectric constant of the environment.

It should be noted that the problem of the nonlinear Schrödinger equation of type (2) is quite general; the treatment of the interaction of a molecule with its environment represents only a special application. Recently, for example, Gisin ${ }^{6,7}$ has used the master equation formalism to develop nonlinear dissipative Schrödinger-like equations for a large class of problems with timeindependent Hamiltonians that involve friction. The Gisin-like equation has been recently reviewed by one of us. $^{8}$

The nonlinear dissipative quantum evolution equation proposed by Gisin has the following simple form: $:^{6,8}$

$$
\frac{d \psi(t)}{d t}=-i \hat{H} \psi(t)+k\left(\langle\hat{H}\rangle_{t}-\hat{H}\right) \psi(t),
$$

where 


$$
\langle\hat{H}\rangle_{t}=\langle\psi(t)|\hat{H}| \psi(t)\rangle .
$$

$\hat{H}$ is the time-independent Hamiltonian and $k(>0)$ is the "quantum" friction constant characterizing the strength of the system-bath interaction. This deterministic nonunitary friction term is motivated by a natural generalization of Wigner's theorem or, equivalently, of Dirac's superposition principle. ${ }^{7}$ It can be applied to any spins as well as to spatial degrees of freedom (or to any quantum system described by a Hilbert space). An example of the classical counterpart of this quantum friction term is the Landau-Lifshitz frictional resistance in the motion of classical spins. ${ }^{9}$ The difference of the Gisin-like formalism with the traditional approaches, such as the Pauli master equation, arises from the fact that in the former case the reduction of the system-bath couplings is done by a pure state preserving projection, ${ }^{6}$ in opposition to the conventional partial trace projection. Thus the nonunitary evolution described by Eq. (3) has the merit that it preserves the superposition principle. Gisin found that Eq. (3) has the unique formal solution

$$
|\psi(t)\rangle=\frac{\exp [-(i+k) \hat{H} t]|\psi(0)\rangle}{[\langle\psi(0)|\exp (-2 k \hat{H} t)| \psi(0)\rangle]^{1 / 2}} .
$$

Two important properties of Eq. (3) are the following: (a) The norm of $\psi(t)$ is conserved even though the system is dissipative, and (b) the following relationship holds true:

$$
\frac{d\langle H\rangle_{t}}{d t}=-2 k\left(\left\langle H^{2}\right\rangle_{t}-\langle H\rangle_{t}^{2}\right) \leq 0 .
$$

Gisin has applied this formalism to spin systems in a magnetic field and to the motion of damped harmonic oscillators. ${ }^{6,7}$

In this paper, we shall explore the detailed nonlinear dynamics for dissipative quantum systems based on an extension of Eq. (3) to the time-periodic Hamiltonian $H(t)=H(t+2 \pi / \omega)$. As will be shown in Secs. II and III, the solution of the Gisin-like equation, Eq. (3), with $\hat{H}(t)$ periodic in time, can be facilitated by the use of the time-independent Floquet matrix method ${ }^{10-12}$ and the generalized Van Vleck nearly degenerate perturbation theory. ${ }^{13,14}$ It is found that $\psi(t)$ approaches asymptotically to a limit cycle with orientation subject to quantum friction constraint. In Sec. IV, we present a detailed analysis of the dissipative dynamics in terms of the trajectory of the Bloch vector. The structure of the limit cycle is found to be (field) frequency and intensity dependent and exhibits an inherent dynamic symmetry. The origin and the role of this dynamical symmetry is analyzed in Sec. V. This is followed by a discussion and conclusion in Sec. VI.

\section{FLOQUET TREATMENT OF NONLINEAR SCHRÖDINGER EQUATION IN PERIODIC FIELDS}

For a time-periodic Hamiltonian, $\hat{H}(t)=\hat{H}(t+T)$, Eq. (3) can be transformed into the following form:

$$
\begin{aligned}
& \frac{d|\Phi(t)\rangle}{d t}=-i \hat{Q}|\Phi(t)\rangle \\
&+k\left[\left\langle\Phi(t)\left|\hat{P}^{+}(t) \hat{H}(t) \hat{P}(t)\right| \Phi(t)\right\rangle\right. \\
&\left.-\hat{P}^{+}(t) \hat{H}(t) \hat{P}(t)\right]|\Phi(t)\rangle,
\end{aligned}
$$

where

$$
|\Phi(t)\rangle=\hat{P}^{+}(t)|\psi(t)\rangle,
$$

and the periodic operator $\widehat{P}(t)$ satisfies the equation ${ }^{11,12}$

$$
\left(\hat{H}(t)-i \frac{d}{d t}\right) \hat{P}(t)=\hat{P}(t) \hat{Q},
$$

with $\hat{Q}$ being the quasienergy operator. Equation (9) can be transformed into an equivalent infinite dimensional time-independent matrix eigenvalue problem, ${ }^{10-12}$

$$
\sum_{\beta, m}\left\langle\alpha n\left|\hat{H}_{F}\right| \beta m\right\rangle P_{\beta k}^{m}=q_{k} P_{\alpha k}^{n},
$$

where $\hat{H}_{F}$ is the time-independent Floquet Hamiltonian defined in the Floquet-state basis $|\alpha n\rangle=|\alpha\rangle \times|n\rangle$,

$$
\left\langle\alpha n\left|\hat{H}_{F}\right| \beta m\right\rangle=H_{\alpha \beta}^{[n-m]}+n \omega \delta_{\alpha \beta} \delta_{n m} .
$$

Here $\{|\alpha\rangle,|\beta\rangle, \ldots\}$ are eigenstates of the unperturbed system, $n$ and $m$ are the Fourier indices (ranging from $-\infty$ to $+\infty), \omega$ is the field frequency, and $k$ is the index of quasienergy states in the reduced zone $(n=m=0)$. In Eq. (11), $H_{\alpha \beta}^{[n]}$ is obtained from the Fourier expansion

$$
\langle\alpha|\hat{H}(t)| \beta\rangle=\sum_{n=-\infty}^{\infty} H_{\alpha \beta}^{[n]} e^{i n \omega t} .
$$

The eigenvector associated with the quasienergy eigenvalue $q_{k n}\left(\equiv q_{k}+n \omega\right)$, denoted by $\left|\lambda_{k n}\right\rangle$, has components defined by

$$
P_{\beta k}^{m}=\left\langle\beta m \mid \lambda_{k 0}\right\rangle,
$$

and satisfying the following periodicity relation:

$$
\left\langle\beta, m+l \mid \lambda_{k, n+l}\right\rangle=\left\langle\beta, m \mid \lambda_{k n}\right\rangle .
$$

Equation (7) can be further transformed into

$$
\frac{d|\chi(t)\rangle}{d t}=k[\langle\chi(t)|\widehat{B}(t)| \chi(t)\rangle-\widehat{B}(t)]|\chi(t)\rangle,
$$

where

$$
|\chi(t)\rangle=e^{i \hat{Q} t}|\Phi(t)\rangle
$$

and

$$
\widehat{B}(t)=e^{i \hat{Q} t} \hat{P}^{+}(t) \hat{H}(t) \hat{P}(t) e^{-i \hat{Q} t} .
$$

In the quasienergy state basis $\left\{\left|\lambda_{k 0}\right\rangle\right\}$, the matrix elements of $\hat{B}(t)$ are

$$
\begin{array}{r}
B_{k k^{\prime}}(t)=\sum_{\alpha, \beta} \sum_{l, n, m} H_{\alpha \beta}^{[l]}\left\langle\lambda_{k 0} \mid \alpha n\right\rangle\left\langle\beta m \mid \lambda_{k^{\prime} 0}\right\rangle \\
\times e^{i(l+m-n) \omega t} e^{i\left(q_{k}-q_{k^{\prime}}\right) t},
\end{array}
$$

where we have used the relation

$$
P_{\alpha k}(t)=\sum_{n} P_{\alpha k}^{n} e^{i n \omega t}=\sum_{n}\left\langle\alpha n \mid \lambda_{k 0}\right\rangle e^{i n \omega t} .
$$

Up to now, no approximation has been made. Equation (14) possesses no closed-form solution. However, an ap- 
proximate analytic solution can be obtained if we take a long-time average on $\widehat{B}(t)$,

$$
\bar{B}_{k k^{\prime}}=\sum_{\alpha, n}\left|\left\langle\alpha n \mid \lambda_{k 0}\right\rangle\right|^{2}\left(q_{k}-n \omega\right) \delta_{k k^{\prime}} .
$$

As will be shown later, this approximation is valid only when the nonlinearity constant $k$ is small. In this case, $[\bar{B}, \hat{Q}]=0$, and the solution of Eq. (3) has the following analytic form:

$$
|\psi(t)\rangle=\widehat{P}(t)\left[e^{-i \hat{Q}_{t}} e^{-k \bar{B}\left(t-t_{0}\right)} e^{i \hat{Q}_{0}} \widehat{P}^{+}\left(t_{0}\right)\left|\psi\left(t_{0}\right)\right\rangle\right]\left[\left\langle\psi\left(t_{0}\right)\left|\widehat{P}\left(t_{0}\right) e^{-i \hat{Q} t_{0}} e^{-2 k \bar{B}\left(t-t_{0}\right)} e^{i \hat{Q}_{0}} \hat{P}^{+}\left(t_{0}\right)\right| \psi\left(t_{0}\right)\right\rangle\right]^{-1 / 2} .
$$

From Eq. (19) we can construct the density matrix

$$
\begin{aligned}
\rho_{\alpha \beta} & \equiv\langle\alpha \mid \psi(t)\rangle\langle\psi(t) \mid \beta\rangle \\
& =\sum_{k, k^{\prime}} \sum_{n, m}\left\langle\alpha n \mid \lambda_{k 0}\right\rangle\left\langle\lambda_{k^{\prime} 0} \mid \beta m\right\rangle e^{-i\left(q_{k}-q_{k^{\prime}}\right) t} e^{i(n-m) \omega t} e^{-k\left(b_{k}+b_{k^{\prime}}\right) t} C_{k} C_{k^{\prime}}^{*} /\left(\sum_{k}\left|C_{k}\right|^{2} e^{-2 k b_{k} t}\right),
\end{aligned}
$$

where we have set $t_{0}=0$ and $b_{k}=\bar{B}_{k k}$. The coefficient $C_{k}$ is given by

$$
C_{k}=\sum_{\alpha, n}\left\langle\lambda_{k 0} \mid \alpha n\right\rangle\langle\alpha \mid \psi(0)\rangle
$$

For near resonance processes, further simplification of Eqs. (10) and (11) is possible by invoking the use of the nearly degenerate perturbation theory. ${ }^{13,14}$ This will be treated in Sec. III.

\section{NEARLY DEGENERATE PERTURBATIVE TREATMENT OF NONLINEAR SCHRÖDINGER EQUATION}

Consider the Gisin-like nonlinear Schrödinger equation in Eq. (3) for a two-level system with the periodic Hamiltonian given by

$$
\hat{H}(t)=\hat{H}_{0}-\mu \cdot \varepsilon \cos (\omega t)
$$

where $\hat{H}_{0}$ is the unperturbed Hamiltonian with eigenvalues $\left(E_{\alpha}=-\omega_{0} / 2, E_{\beta}=\omega_{0} / 2\right)$ and eigenvectors $(|\alpha\rangle$, $|\beta\rangle) ; \mu$ is the dipole moment and $\varepsilon$ the electric field amplitude of the laser field. $\hat{H}(t)$ can be rewritten, in matrix form, as

$$
\hat{H}(t)=\left[\begin{array}{cc}
-\omega_{0} / 2 & 2 \lambda \cos (\omega t) \\
2 \lambda \cos (\omega t) & \omega_{0} / 2
\end{array}\right],
$$

where $\lambda$ is the coupling strength

$$
\lambda=-\frac{1}{2}\langle\alpha|\mu \cdot \varepsilon| \beta\rangle \text {. }
$$

Resonant transition between $|\alpha\rangle$ and $|\beta\rangle$ occurs whenever $\omega_{0}=E_{\beta}-E_{\alpha} \cong(2 n+1) \omega, n=0,1,2, \ldots$. In this case, the nearly degenerate perturbation method such as the generalized Van Vleck (GVV) theory ${ }^{13,14}$ can be employed to reduce the infinite dimensional Floquet matrix $\hat{H}_{F}$, Eq. (11), into a two-by-two time-independent effective matrix. In addition, the GVV method has the merit that if the wave function is accurate to the $m$ th order, the corresponding eigenvalue will be accurate to the $(2 m+1)$ th order. Thus, for example, to the first order in wave function and to the third order in quasienergy, the GVV Hamiltonian has the form (when $\omega_{0} \cong \omega$ )

$$
H_{\mathrm{GVV}}=\left[\begin{array}{cc}
-\frac{\omega_{0}}{2}-\frac{\lambda^{2}}{\omega+\omega_{0}} & \lambda-\frac{\lambda^{3}}{\left(\omega+\omega_{0}\right)^{2}} \\
\lambda-\frac{\lambda^{3}}{\left(\omega+\omega_{0}\right)^{2}} & \frac{\omega_{0}}{2}-\omega+\frac{\lambda^{2}}{\omega+\omega_{0}}
\end{array}\right] .
$$

Similar GVV Hamiltonians can be constructed for multiphoton processes. ${ }^{13,14}$ The GVV quasienergies $q_{ \pm}$can be readily obtained from $\mathrm{Eq}$. (24),

$$
q_{ \pm}=-\frac{\omega}{2} \pm \Omega
$$

where $\Omega$ is the Rabi frequency given by

$$
\Omega=\left[\Delta^{2}+\left[\lambda-\frac{\lambda^{3}}{\left(\omega+\omega_{0}\right)^{2}}\right]^{2}\right]^{1 / 2}
$$

and $\Delta$ is the detuning parameter,

$$
\Delta=\frac{1}{2}\left(\omega-\omega_{0}\right)-\lambda^{2} /\left(\omega_{0}+\omega\right) .
$$

The long-time average of $\hat{B}$, Eq. (19), in the GVV approximation reduces to $\left(b_{+} \equiv \bar{B}_{++}, b_{-} \equiv \bar{B}_{--}\right)$,

$$
\begin{aligned}
b_{+}=-b_{-}= & \left\{\Omega-\frac{\omega \Delta}{2 \Omega}\left[1+2\left[\frac{\lambda}{\omega+\omega_{0}}\right]^{2}\right]\right. \\
& \times\left[1+\left(\frac{\lambda}{\omega+\omega_{0}}\right]^{2}\right]^{-1} .
\end{aligned}
$$

It is interesting to note that there exists a field frequency $\omega=\omega_{c}$ at which $b_{+}=b_{-}=0$ and the effect of quantum friction disappears. Figure 1(a) shows the time-average transition probability $\bar{P}_{\alpha \rightarrow \beta}$ as a function of $\omega_{0} / \omega$ for a two-level system with the physical parameters set at $\omega_{0}=1.0, k=0.01$, and $\lambda=0.1 \omega$ (arbitrary units). Both the one- and three-photon transitions are shown. The most distinctive feature is that $\bar{P}_{\alpha \rightarrow \beta}$ can exceed 0.5 (i.e., antipopulation). This is contrary to the nondissipative case $(k=0)$, where $\bar{P}_{\alpha \rightarrow \beta} \leq \frac{1}{2}$. Figure 1(b) shows the behavior of $\bar{B}_{++}\left(b_{+}\right)$and $\bar{B}_{--}\left(b_{-}\right)$as a function of $\omega_{0} / \omega$. Notice that $b_{+}$and $b_{-}$cross each other every time there is a resonance transition between the two levels. However, the crossing points (at which $b_{+}=b_{-}=0$ ) need not coincide with the resonance frequencies (at which $\bar{P}_{\alpha \rightarrow \beta}$ 
reach maxima).

The dynamics of the two-level system can be represented by the rotation of the Bloch vector $\mathbf{S}=\left(u^{\prime}, v^{\prime}, w^{\prime}\right)$ in three-dimensional space in the laboratory frame, where

$$
\begin{aligned}
& u^{\prime}=\left(\rho_{\alpha \beta}+\rho_{\beta \alpha}\right), \\
& v^{\prime}=-i\left(\rho_{\alpha \beta}-\rho_{\beta \alpha}\right),
\end{aligned}
$$

and

$$
w^{\prime}=\rho_{\beta \beta}-\rho_{\alpha \alpha} .
$$

It is often convenient to work in the rotating frame described by the transformation

$$
\left(\begin{array}{l}
u \\
v \\
w
\end{array}\right)=\left(\begin{array}{ccc}
\cos (\omega t) & \sin (\omega t) & 0 \\
-\sin (\omega t) & \cos (\omega t) & 0 \\
0 & 0 & 1
\end{array}\right)\left(\begin{array}{l}
u^{\prime} \\
v^{\prime} \\
w^{\prime}
\end{array}\right) .
$$

In the following, we shall first discuss the nonlinear dynamics of the dissipative two-level system when the nonlinearity $k$ is small. In this case, the long-time average of the operator $\hat{B}(t)$ is a valid approximation, and an approximate analytic solution can be derived for the Bloch vector $\mathbf{S}(t)$.

\section{A. Dissipative nonlinear dynamics in the case of small} nonlinearity (friction) - long-time average approximation

The components of the Bloch vector $\mathbf{S}(t)$, in the rotating frame, can be obtained analytically in the GVV approximation if the long-time average is performed for the operator $\hat{B}(t)$ before Eq. (14) is solved. For the onephoton dominant frequency region $\left(\omega \sim \omega_{0}\right)$, for example, we obtain
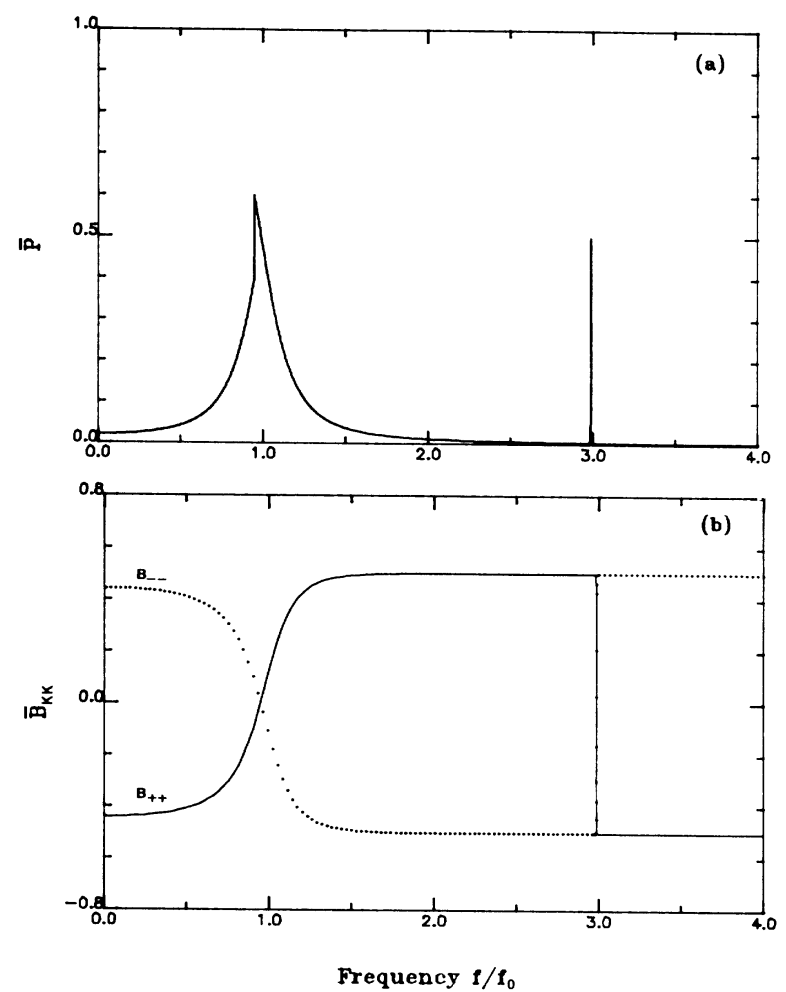

FIG. 1. (a) Time-average transition probability $\bar{P}_{\alpha+\beta}$ and (b) $\bar{B}_{\ldots} \quad$ (dotted line) and $\bar{B}_{++}$(solid line) as a function of $\omega_{0} / \omega$ for a dissipative two-level system $\left(f / f_{0}=\omega_{0} / \omega\right)$. The physical parameters used are $\omega_{0}=1.0, k=0.01$, and $\lambda=0.1 \omega$ (arbitrary units).

$$
\begin{aligned}
& u(t)=A\left\{\left[\sin (2 \theta)-\frac{2 \lambda}{\left(\omega+\omega_{0}\right)} \cos (2 \theta) \cos (2 \omega t)-\left(\frac{\lambda}{\omega+\omega_{0}}\right)^{2} \sin (2 \theta) \cos (4 \omega t)\right] B\right. \\
& +2\left[\cos (2 \theta) \cos (2 \Omega t)+\frac{\lambda}{\left(\omega+\omega_{0}\right)} \sin (2 \theta) \cos (2 \omega t) \cos (2 \Omega t)-\left(\frac{\lambda}{\omega+\omega_{0}}\right)^{2} \cos (2 \theta) \cos (4 \omega t) \cos (2 \Omega t)\right. \\
& \left.\left.-\left(\frac{\lambda}{\omega+\omega_{0}}\right)^{2} \sin (4 \omega t) \sin (2 \Omega t)\right] C_{+} C_{-}\right\} / D \\
& v(t)=A\left\{\left[\frac{2 \lambda}{\omega+\omega_{0}} \cos (2 \theta) \sin (2 \omega t)+\left[\frac{\lambda}{\omega+\omega_{0}}\right]^{2} \sin (2 \theta) \sin (4 \omega t)\right] B\right. \\
& -2\left[\sin (2 \Omega t)+\left(\frac{\lambda}{\omega+\omega_{0}}\right) \sin (2 \theta) \sin (2 \omega t) \cos (2 \Omega t)-\left(\frac{\lambda}{\omega+\omega_{0}}\right]^{2} \cos (2 \theta) \sin (4 \omega t) \cos (2 \Omega t)\right. \\
& \left.\left.-\left(\frac{\lambda}{\omega+\omega_{0}}\right)^{2} \cos (4 \omega t) \sin (2 \Omega t)\right] C_{+} C_{-}\right\} / D \\
& w(t)=A\left[\left\{\cos (2 \theta)\left[1-\left(\frac{\lambda}{\omega+\omega_{0}}\right]^{2}\right]+\frac{2 \lambda}{\omega+\omega_{0}} \sin (2 \theta) \cos (2 \omega t)\right\} B\right. \\
& -2\left\{\sin (2 \theta)\left[1-\left[\frac{\lambda}{\omega+\omega_{0}}\right]^{2}\right] \cos (2 \Omega t)\right. \\
& \left.\left.-\frac{2 \lambda}{\omega+\omega_{0}}[\cos (2 \theta) \cos (2 \Omega t) \cos (2 \omega t)+\sin (2 \Omega t) \sin (2 \omega t)]\right\} C_{+} C_{-}\right] D \text {, }
\end{aligned}
$$


where

$$
\begin{aligned}
& A=\left[1+\left[\frac{\lambda}{\omega+\omega_{0}}\right]^{2}\right]^{-1}, \quad B=C_{+}^{2} e^{-2 k b t}-C_{-}^{2} e^{2 k b t}, \quad C_{+}=[(\Omega+\Delta) / 2 \Omega]^{1 / 2}, \\
& C_{-}=-\lambda[2 \Omega(\Omega+\Delta)]^{-1 / 2}, \quad D=C_{+}^{2} e^{-2 k b t}+C_{-}^{2} e^{2 k b t}, \quad b=b_{+}=-b_{-},
\end{aligned}
$$

and

$$
\cos \theta=[(\Omega+\Delta) / 2 \Omega]^{1 / 2}
$$

From Eqs. (30a)-(30c), we see that after some long time, $t \gg 1 / 2 k b$, the Bloch vector will settle down to a simple precession around a fixed vector (limit cycle). Assuming $b>0$, we have asymptotically

$$
\begin{aligned}
& u(t)=-\left[1+\left[\frac{\lambda}{\omega+\omega_{0}}\right]^{2}\right]^{-1}\left\{\left[\sin (2 \theta)-\frac{2 \lambda}{\omega+\omega_{0}} \cos (2 \theta) \cos (2 \omega t)-\left(\frac{\lambda}{\omega+\omega_{0}}\right]^{2} \sin (2 \theta) \cos (4 \omega t)\right],\right. \\
& v(t)=-\left[1+\left(\frac{\lambda}{\omega+\omega_{0}}\right)^{2}\right]^{-1}\left[\frac{2 \lambda}{\omega+\omega_{0}} \cos (2 \theta) \sin (2 \omega t)+\left(\frac{\lambda}{\omega+\omega_{0}}\right)^{2} \sin (2 \theta) \sin (4 \omega t)\right] \\
& w(t)=-\left[1+\left(\frac{\lambda}{\omega+\omega_{0}}\right)^{2}\right]^{-1}\left[\left(1-\frac{\lambda^{2}}{\left(\omega+\omega_{0}\right)^{2}}\right) \cos (2 \theta)+\frac{2 \lambda}{\omega+\omega_{0}} \sin (2 \theta) \cos (2 \omega t)\right] .
\end{aligned}
$$

Equations (31a)-(31c) show that the limit cycle structure is $k$ independent if a long-time average approximation is made for $\hat{B}(t)$. Further, the Rabi-frequency $(\Omega)$ contributions to $\mathbf{S}(t)$ decay out completely after $\mathbf{S}(t)$ settles down to the limit cycle.

Figure 2 shows the time evolution of the Bloch vector in the $w-v$ plane for three different time intervals (in field oscillations, $T=2 \pi / \omega)$ : (a) $[0,50 T]$, (b) $[600 T, 650 T]$, and (c) $[5000 T, 5050 T]$. The physical parameters used are $\omega_{0}=1, \omega=1.0390 \omega_{0}, \lambda=0.1 \omega$, and $k=0.01$. It is seen that the Bloch vector components exhibit quasiperiodic and shrinking motion simultaneously and reach

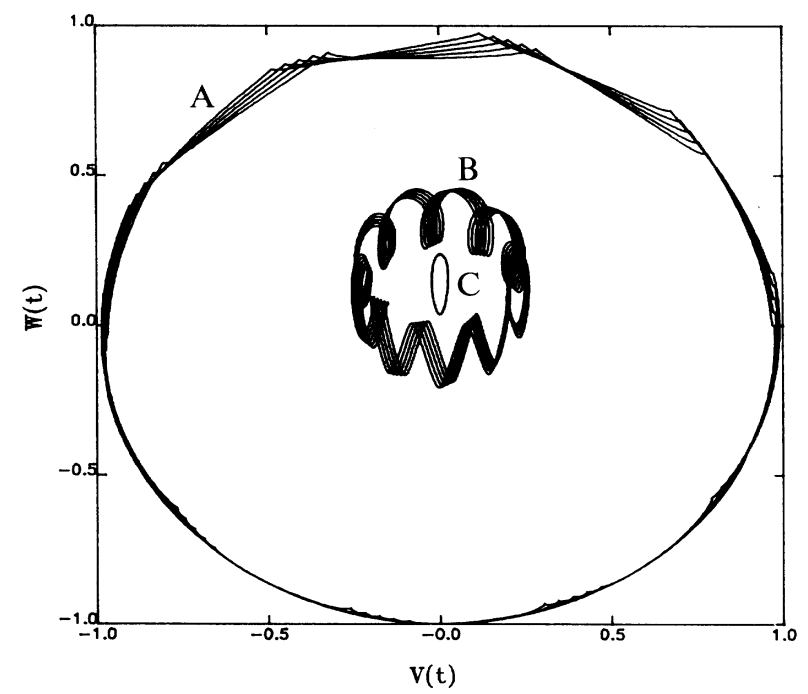

FIG. 2. Time evolution of the Bloch vector $\mathbf{S}(t)$ in the $w-v$ plane. The trajectories labeled $A, B$, and $C$ correspond to the time intervals in $[0,50 T],[600 T, 650 T]$, and [5000T,5050T], respectively, where $T=2 \pi / \omega$ is the period of field oscillations. Physical parameters same as Fig. 1 except $\omega / \omega_{0}=1.0395$. a limit cycle asymptotically. Similar phenomena are also observed in the $w-u$ and $u-v$ planes.

\section{B. RWA analytic solution}

In this section we show that the Gisin-like equation, Eq. (3), with $\hat{H}(t)$ periodic in time can also be solved analytically in closed form within the rotating-wave approximation (RWA) without the need of using the longtime average approximation for $\widehat{B}(t)$. Under the RWA, the total Hamiltonian $\hat{H}(t)$, Eq. (24), is replaced by

$$
\hat{H}_{\mathrm{RWA}}(t)=\left[\begin{array}{ll}
-\omega_{0} / 2 & b e^{i \omega t} \\
b e^{-i \omega t} & \omega_{0} / 2
\end{array}\right],
$$

where $b=-\langle\alpha|\boldsymbol{\mu} \cdot \varepsilon| \beta\rangle / 2$ is the coupling strength. We now consider the solution of the transformed equation, Eq. (7). The periodic operator $\widehat{P}(t)$ allows the transformation of $\widehat{H}_{\mathrm{RWA}}(t)$ into a time-independent matrix $\widetilde{B}$,

$$
\widetilde{B}=\widehat{P}^{+}(t) \hat{H}_{\mathrm{RWA}}(t) \hat{P}(t) .
$$

To see this we need to first work out $\widehat{P}(t)$. In RWA, the quasienergy eigenvalues $q_{ \pm}$can be obtained easily as

$$
q_{ \pm}=-\omega / 2 \pm \Omega \text {, }
$$

where

$$
\Omega=\left(b^{2}+\Delta^{2}\right)^{1 / 2}
$$

and

$$
\Delta=\left(\omega-\omega_{0}\right) / 2
$$

The corresponding eigenvectors are

$$
\begin{aligned}
& \left|\lambda_{+0}\right\rangle=(\cos \theta)|\alpha, 0\rangle+(\sin \theta)|\beta,-1\rangle, \\
& \left|\lambda_{-0}\right\rangle=-(\sin \theta)|\alpha, 0\rangle+(\cos \theta)|\beta,-1\rangle,
\end{aligned}
$$

where $|\alpha, 0\rangle$ and $|\beta,-1\rangle$ are the two unperturbed Floquet basis states, and 


$$
\cos \theta=[(\Omega+\Delta) / 2 \Omega]^{1 / 2} .
$$

The matrix elements $P_{\gamma k}(t)$ ( $\gamma=\alpha$ or $\beta, k=+$ or - ) can now be constructed according to Eq. (18),

$$
P_{\gamma k}(t)=\sum_{n}\left\langle\gamma n \mid \lambda_{k 0}\right\rangle e^{i n \omega t}
$$

$$
\widetilde{B}=\left[B_{k k^{\prime}}\right]=\left[\begin{array}{cc}
\left(-\omega_{0} / 2\right) \cos (2 \theta)+b \sin (2 \theta) & \left(\omega_{0} / 2\right) \sin (2 \theta)+b \cos (2 \theta) \\
\left(\omega_{0} / 2\right) \sin (2 \theta)+b \cos (2 \theta) & \left(\omega_{0} / 2\right) \cos (2 \theta)-b \sin (2 \theta)
\end{array}\right],
$$

which is indeed time independent. Equation (7) now becomes

$$
d|\Phi(t)\rangle / d t=-i \hat{Q}|\Phi(t)\rangle+k[\langle\Phi(t)|\widetilde{B}| \Phi(t)\rangle-\widetilde{B}] \Phi(t),
$$

which allows for a closed-form solution similar to that in Eq. (5). Using the relation in Eq. (8),

$$
|\psi(t)\rangle=\hat{P}(t)|\Phi(t)\rangle
$$

we arrive at the desired RWA solution

$$
|\psi(t)\rangle_{\mathrm{RWA}}=\hat{P}(t) \frac{e^{i(\hat{Q}-i k \tilde{B}) t} \hat{P}+(0)|\psi(0)\rangle}{\left[\left\langle\psi(0)\left|\hat{P}(0) e^{i(\hat{Q}+i k \tilde{B}) t} e^{-i(\hat{Q}-i k \tilde{B})} \hat{P}+(0)\right| \psi(0)\right\rangle\right]^{1 / 2}} .
$$

Note that the quasienergy operator $\hat{Q}$ is diagonal in the $\left|\lambda_{ \pm 0}\right\rangle$ basis. That is,

$$
\hat{Q}=\left[\begin{array}{cc}
q_{+} & 0 \\
0 & q_{-}
\end{array}\right]
$$

Further, $\hat{Q}$ and $\widetilde{B}$ do not commute. The operator $\widetilde{Q} \equiv \widehat{Q}-i k \widetilde{B}$ is non-Hermitian and possesses complex eigenvalues. Care should be taken to obtain correct eigensolutions by using the biorthonormal relationships. ${ }^{12}$ It can be shown ${ }^{15}$ that in the rotating frame, Eq. (29), the RWA Bloch vector $\mathbf{S}_{\mathrm{RWA}}=(u, v, w)$ reaches asymptotically to a (time-independent) fixed vector rather than a limit cycle. The RWA solution is valid, in general, only for weak-field and near resonant one-photon transition processes. For stronger fields and/or multiphoton processes, higher-order GVV and/or numerical methods are required to obtain the correct solutions as described in Sec. IV.

\section{DISSIPATIVE NONLINEAR QUANTUM DYNAMICS IN TWO-LEVEL SYSTEMS - NUMERICAL SOLUTIONS}

In this section we present a detailed study of the dissipative nonlinear dynamics of a two-level system perturbed by periodic fields. Particular attention will be paid to the (field) frequency- and intensity-dependent dissipative dynamical evolution and the structure of the limit cycle. When $k$ is not small or the field intensity strong, the long-time average or the RWA solution described in Sec. III is not valid and numerical techniques such as the Runge-Kutta method will be used for the solution of Eq. (14). For nearly resonant multiphoton processes, the operator $\hat{B}(t)$ defined in Eq. (17) can be simplified by means of the high-order GVV approximation of the Floquet Hamiltonian $\hat{H}_{F}$.

Since the norm of the wave function $\psi(t)$ is conserved in time, the length of the Bloch vector $\mathbf{S}(t)$ is invariant in the rotating frame, namely,

$$
u^{2}(t)+v^{2}(t)+w^{2}(t)=1 .
$$

Thus only two independent variables are required to specify the motion of the Bloch vector. We shall choose the population difference $w\left(=\rho_{\beta \beta}-\rho_{\alpha \alpha}\right)$, and the angle

$$
\phi=\cos ^{-1}\left[u /\left(u^{2}+v^{2}\right)^{1 / 2}\right]
$$

as our variables.

Figure 3 shows a typical trajectory in the $\phi-w$ plane from $t=0$ to $100 T(T=2 \pi / \omega)$. The physical parameters used are $\omega_{0}=1.0, \omega=1.0395 \omega_{0}, \lambda=0.1 \omega$, and $k=0.165$ (arbitrary units). Only the points at each time interval

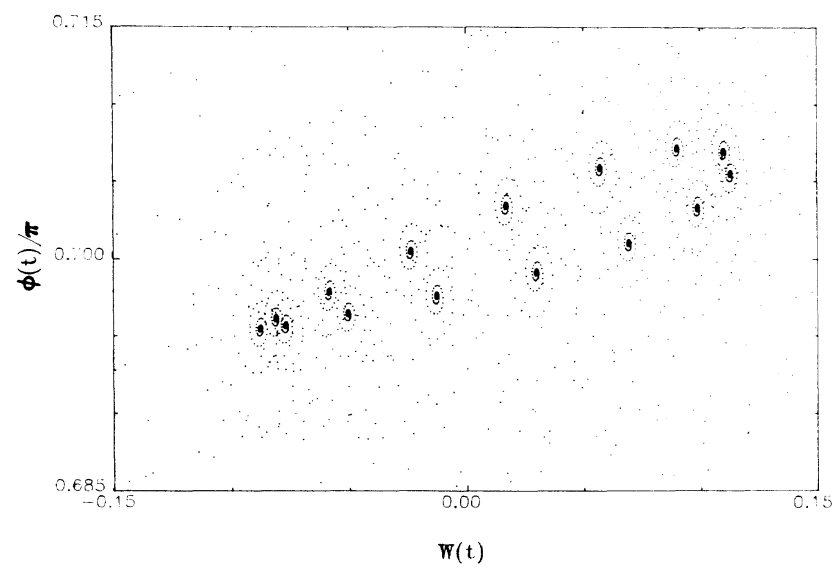

FIG. 3. Typical one-photon dominant Bloch vector trajectory in the $\phi-w$ plane from $t=0$ to $100 T$, where the angle $\phi$ is defined in Eq. (44). The trajectory points are recorded at every $\Delta t=2 \pi / 30 \omega$. The physical parameters used are $\omega_{0}=1.0$, $\omega=1.0395 \omega_{0}, \lambda=0.1 \omega$, and $k=0.165$. 
$\Delta t=2 \pi / 30 \omega$ are recorded. This reveals several interesting dynamical features. First there are points scattered over the $\phi-w$ plane. Second, there are 15 point attractors (in the center portion of the graph) forming a limit cyclelike structure. Third, each point attractor is surrounded by a similar spiral substructure. The fact that there are 15 point attractors means that it takes $15 \Delta t=15(2 \pi /$ $30 \omega)=2 \pi / 2 \omega$ amount of time for the system to travel around the limit cycle once. In other words, the system exhibits a $2 \omega$ frequency in its time evolution. This is related to the dynamical symmetry inherent in the system to be discussed in Sec. V.

Figure 4 illustrates yet another striking phenomenon in nonlinear dynamics. Here the time interval is cut finer to $\Delta t=2 \pi / 70 \omega$. We now see 35 point attractors forming the limit cycle; each one of them is again surrounded by similar spiral structure. The number 35 again arises from the $2 \omega$ frequency period. If we cut the time interval finer and finer, we see more and more point attractors. Each one always exhibits self-similar spiral structure. The invariance of this self similarity under changes of magnification suggests the system exhibits fractal-like characteristics during its time evolution. In Fig. 5, we plot the same trajectory data in Fig. 4 but only from $=50 T$ to $100 T(T=2 \pi / \omega)$. We see all the scattered points disappear and only the point attractors and their spiral structures remain. This clearly indicates that the dissipative system evolves asymptotically to a limit cycle, with each point of the limit cycle being a point attractor.

Figure 6 shows another example of the spiral pattern near by the limit cycle for a three-photon dominant process. (The physical parameters used are $\omega=\omega_{0} / 3$, $\omega_{0}=1.0, \lambda=0.1 \omega_{0}$, and $k=0.01$.) In this latter case $k$ is rather small, and Fig. 7 shows the corresponding result generated from the long-time average analytical formula. Apart from a slight shift in the position, the two structures agree rather well, indicating the validity of the long-time average approximation for small nonlinearity cases.

We now discuss the intensity-dependent limit cycle structure. Figures $8-10$ show the limit cycle structures for one-photon dominant processes (at fixed $\omega_{0}=1.0$,

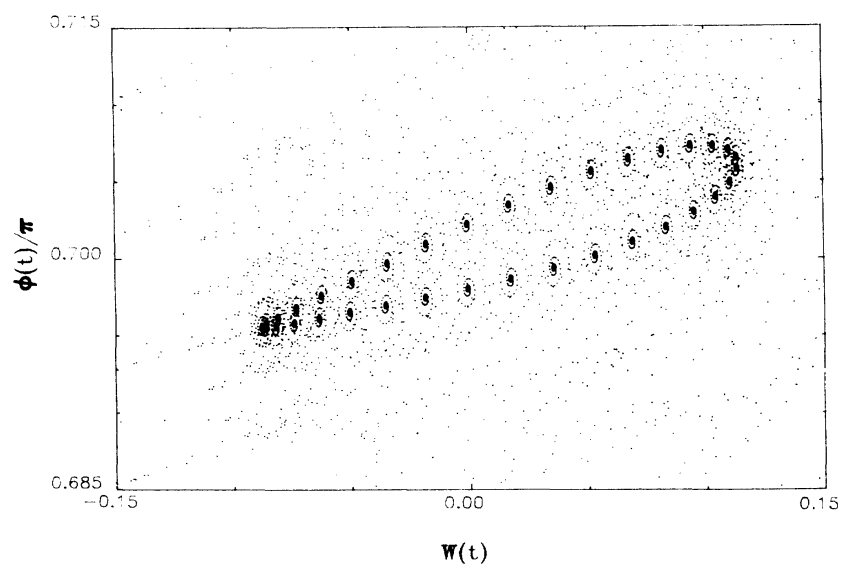

FIG. 4. Same as Fig. 3 except $\Delta t=2 \pi / 70 \omega$.

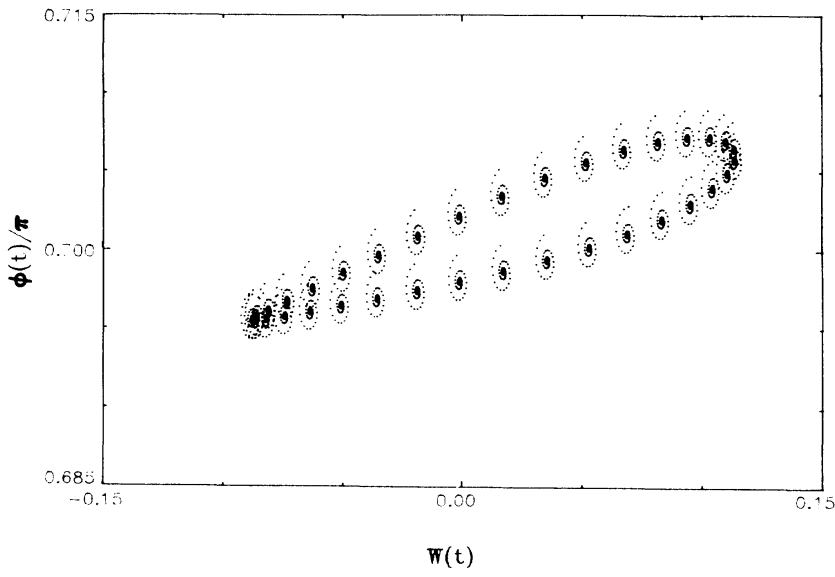

FIG. 5. Same as Fig. 4 except only the data from $t=50 T$ to $100 T$ are recorded. It shows clearly the spiral structure around each point (attractor) of the limit cycle.

$k=0.1$, and $\omega=\omega_{0}$ ) for coupling strengths $\lambda / \omega_{0}=0.1$, 0.5 , and 1.0 , respectively. The main feature changes as $\lambda$ increases are the gradual increase in size of the limit cycle. While the shape of the limit cycle also changes in shape considerably as $\lambda$ varies, it remains a single folded structure. The situation for the multiphoton transition case is more involved. Figures 11-13 depict the limit cycle structures for three-photon dominant processes (at fixed $\omega_{0}=1.0, k=0.1$, and $\omega=\omega_{0} / 3$ ) for $\lambda / \omega_{0}=0.1,0.5$, and 1.0, respectively. In addition to the change in size as $\lambda$ varies, the limit cycles exhibit multifolded structures at high-field intensities. Clearly, the structure of the limit cycle depends upon the order of nonlinear multiphoton transitions. An interesting question now arises: Can the dissipative quantum system exhibit bifurcation or even chaos at very high laser intensities? We have performed several high intensity calculations (up to $\lambda / \omega_{0}=10.0$ ). In each case, while the structure of limit cycle is getting

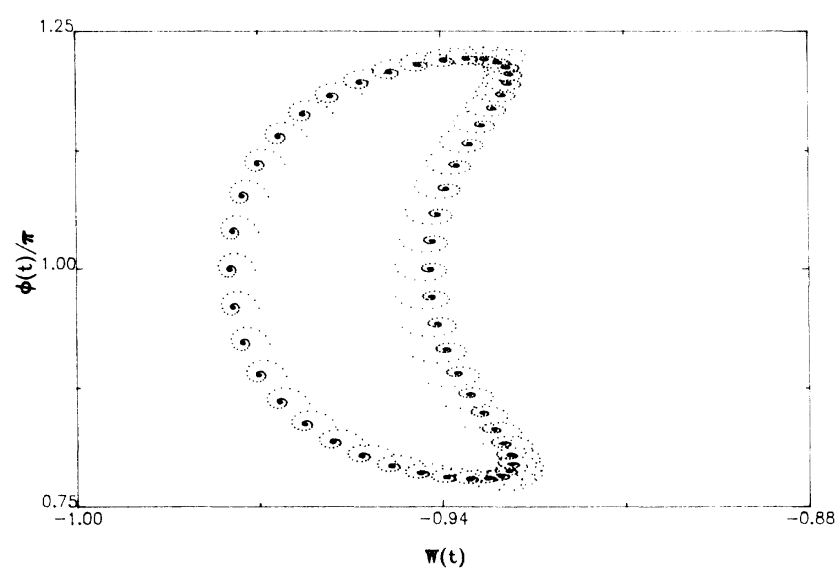

FIG. 6. Spiral pattern nearby the limit cycle for a threephoton dominant process. The results are obtained by "exact" numerical solutions. The physical parameters used are $\omega_{0}=1.0$, $\omega=\omega_{0} / 3, \lambda=0.1 \omega_{0}$, and $k=0.01$. 


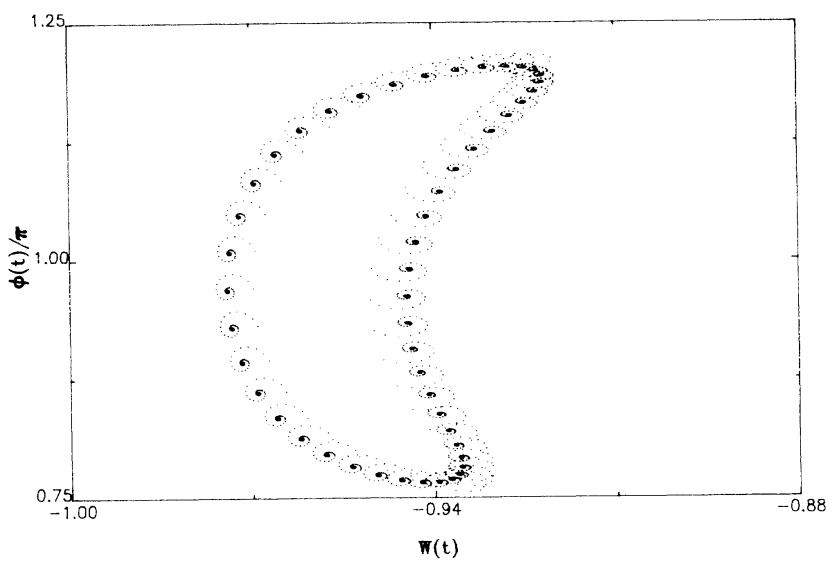

FIG. 7. Same as Fig. 7 except the results are obtained by the long-time average approximation.

more complicated as $\lambda$ increases, it always remains quasiperiodic. Thus for a dissipative quantum system under periodic perturbation, there is no stochastic motion observed. This is an example of the so-called quantum suppression of classical chaos. ${ }^{16}$ While the corresponding classical systems can exhibit chaotic motions, the quantum interference effects tend to suppress the irregular behavior. The absence of "quantum" chaos, however, does not prevent the existence of fractal-like behavior.

\section{DYNAMICAL SYMMETRY OF THE LIMIT CYCLE}

Figure 14 shows the power spectrum

$$
W(f)=\int_{0}^{t_{f}} w\left(t^{\prime}\right) e^{i \omega t^{\prime}} d t^{\prime} \quad(\omega=2 \pi f),
$$

for $w(t)$ in the time interval $(0,100 T)$ for the one-photon dominant process at $\omega_{0}=1.0, \omega=\omega_{0}, k=0.1$, and $\lambda=1.0 \omega_{0}$ (strong-field case). One sees that besides the

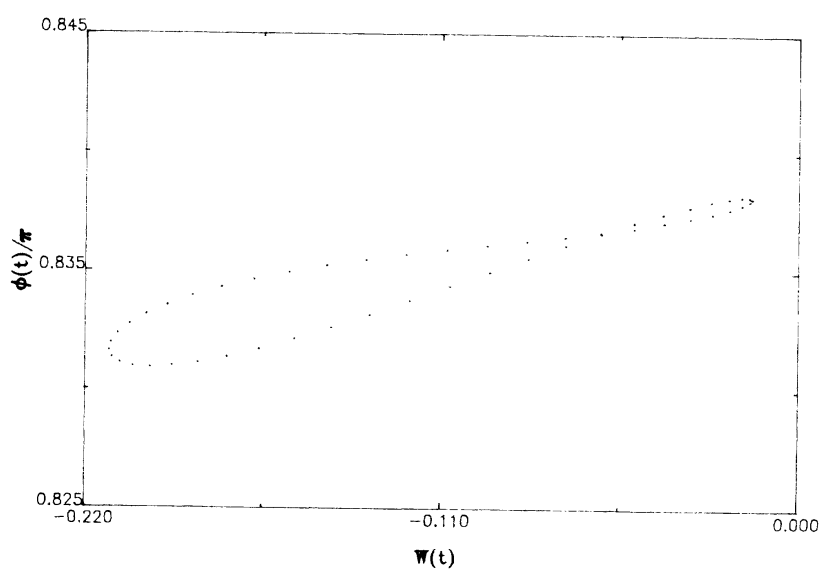

FIG. 8. Asymptotic limit cycle structure for a one-photon dominant process at $\omega_{0}=1.0, k=0.1, \omega=\omega_{0}$, and coupling strength $\lambda=0.1 \omega_{0}$ (weak field).

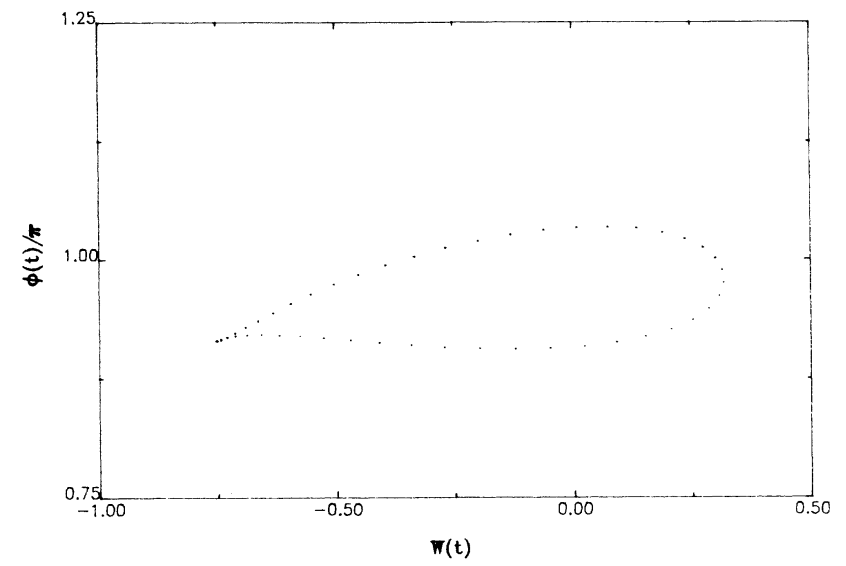

FIG. 9. Same as Fig. 8 except $\lambda=0.5 \omega_{0}$ (medium strong field).

sharp peaks at $2 \omega, 4 \omega, 6 \omega$, and $8 \omega$, etc., there are broader peaks inbetween them. Figure 15 shows the power spectrum for the same process in the longer-time interval $(100 T, 200 T)$. Here all the broadened peaks disappear and only the sharp peaks at $2 n \omega(n=1,2,3,4)$ remain. This corresponds to the power spectrum of the limit cycle shown in Fig. 10. Similar behavior is observed for the three-photon dominant case $\left(\omega_{0}=1.0, \omega=\omega_{0} / 3, \quad \lambda\right.$ $\left.=0.1 \omega_{0}, k=0.01\right)$ as shown in Figs. 16 and 17. One thus sees clearly that there is a $2 \omega$ fundamental frequency inherent in the dissipative dynamics as well as in the limit cycle in the rotating frame of coordinates.

The origin of this fundamental frequency $2 \omega$ can be understood from an inspection of Eqs. (30) and (31). During the earlier dissipative temporal evolution, Eq. (30) shows that $w(t)$ contains two different dynamical components: One is characterized by the $\cos (2 n \omega t)$ [or $\sin (2 n \omega t)]$ oscillations and the other by the Rabi oscillations $[\cos (2 \Omega t)$ or $\sin (2 \Omega t)$ ]. The Rabi oscillation component is associated with the appearance of the broadened peaks during the evolution and gradually decays away. After the sys-

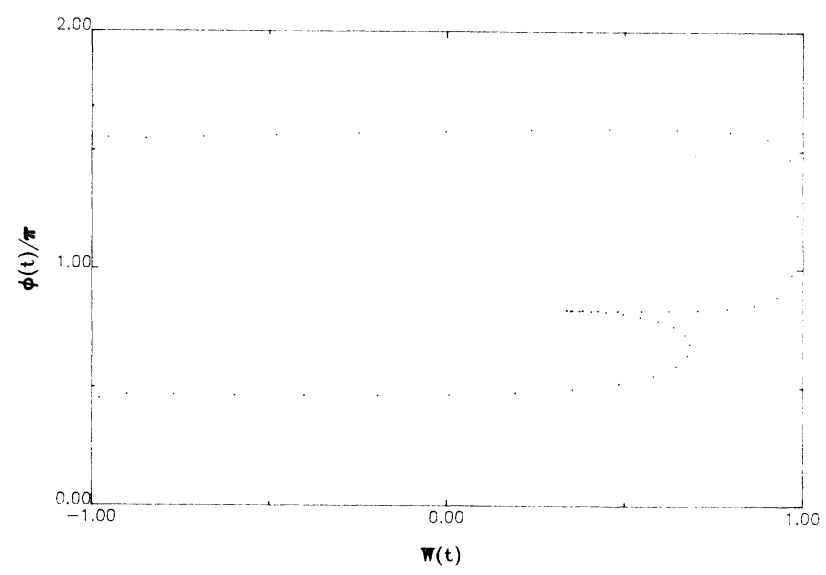

FIG. 10. Same as Fig. 8 except $\lambda=1.0 \omega_{0}$ (strong field). 


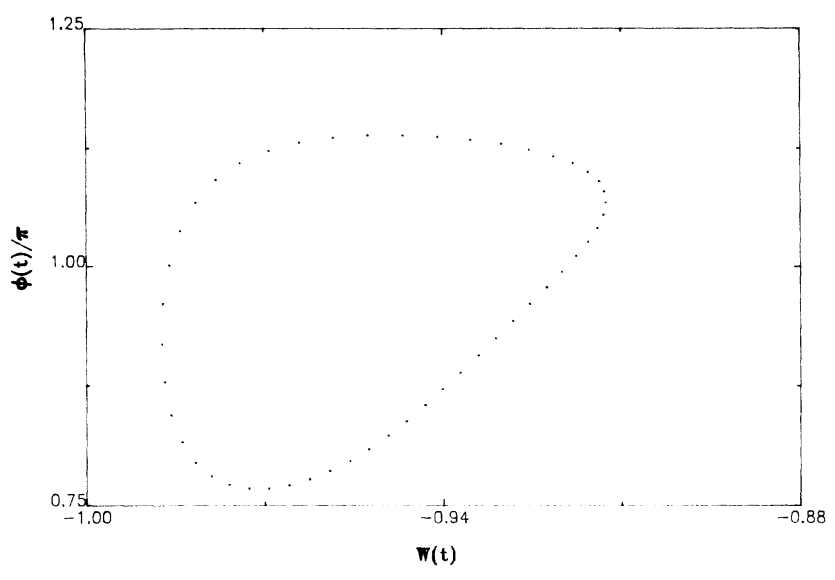

FIG. 11. Asymptotic limit cycle structure for a three-photon dominant process at $\omega_{0}=1.0, k=0.1, \omega=\omega_{0} / 3$, and $\lambda=0.1 \omega_{0}$.

tem settles down to the limit cycle, only the $2 n \omega$ frequency component remains [Eq. (31)].

In classical nonlinear dynamics, a system driven by a periodic force $F(x, t+T)=F(x, t)$ is said to be a symmetric system ${ }^{17}$ if $x(t)$ is a solution so is $-x(t+T / 2)$. We call $x(t)$ a symmetric solution ${ }^{17}$ of period $T$ if $x(t)=-x(t+T / 2)$. The possession of dynamical symmetry leads to the suppression of period doubling and classical chaos. ${ }^{17}$ We now examine whether this idea can be extended to a nonlinear quantum system. The Gisinlike equation, Eq. (3), in density-matrix formulation can be written as

$$
\frac{d}{d t} \hat{\rho}(t)=i[\hat{\rho}(t), \hat{H}(t)]+k[[\hat{\rho}(t), \hat{H}(t)], \hat{\rho}(t)] .
$$

The Hamiltonian in the current study of two-level systems, Eq. (24), possesses the following properties:

$$
\hat{H}(t+T)=\hat{H}(t), \quad T=2 \pi / \omega
$$

and

$$
\hat{H}(t+T / 2)=\widehat{J} \hat{H}(t) \hat{J}^{+},
$$

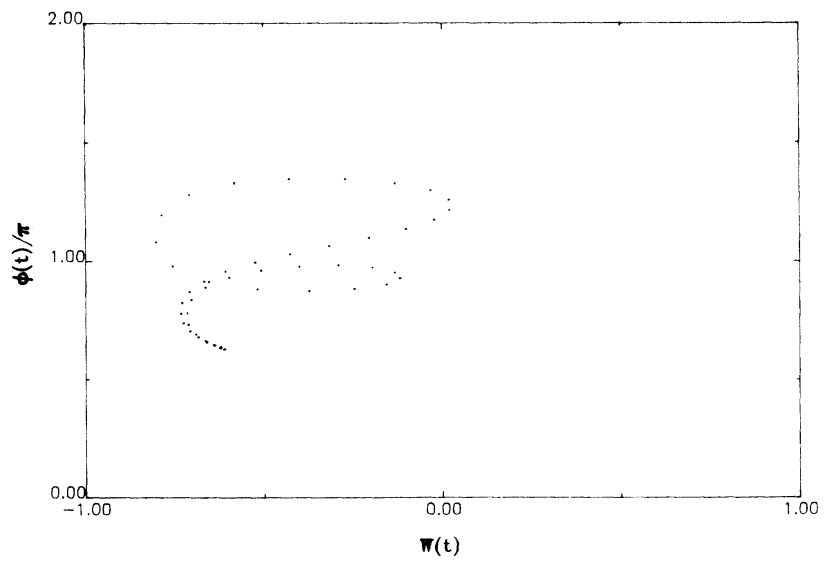

FIG. 12. Same as Fig. 11 except $\lambda=0.5 \omega_{0}$.

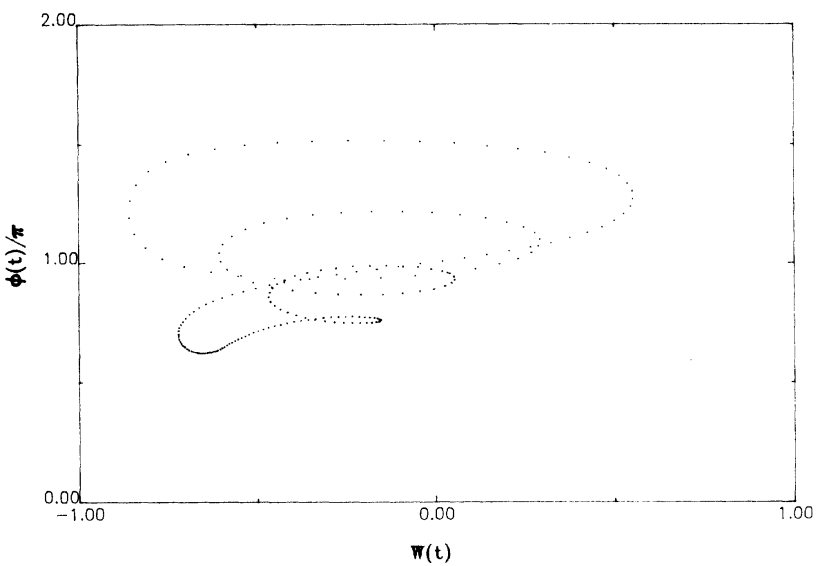

FIG. 13. Same as Fig. 11 except $\lambda=1.0 \omega_{0}$.

where

$$
\hat{J}=\left[\begin{array}{cc}
i & 0 \\
0 & -i
\end{array}\right]=-\widehat{J}^{+} .
$$

It can be shown ${ }^{15}$ that if $\hat{\rho}(t)$ is a solution of Eq. (46), $\hat{J} \hat{\rho}(t+T / 2) \hat{J}^{+}$will also be a solution. In general, $\hat{J} \hat{\rho}(t+T / 2) \hat{J}^{+}$need not be equal to $\hat{\rho}(t)$. Following the classical analog, ${ }^{17}$ we shall call a nonlinear quantum system dynamically symmetric if

$$
\hat{J} \hat{\rho}^{(t+T / 2) \hat{J}^{+}=\hat{\rho}(t) .}
$$

We found this relation is indeed satisfied once the system has reached the limit cycle. Let us analyze the consequence of this equality. In the rotating frame of coordinates, defined in Eq. (29), Eq. (50) leads to

$$
\left[\begin{array}{l}
u(t+T / 2) \\
v(t+T / 2) \\
w(t+T / 2)
\end{array}\right]=\left[\begin{array}{c}
u(t) \\
v(t) \\
w(t)
\end{array}\right) .
$$

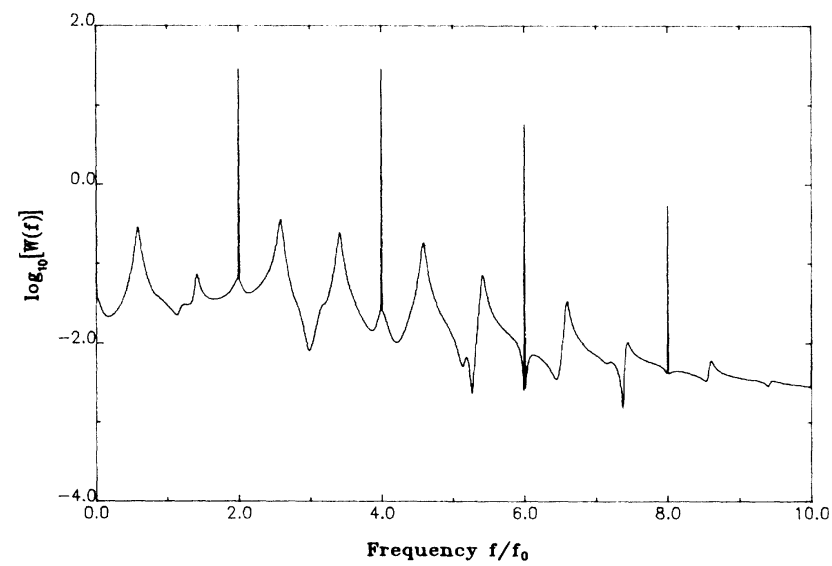

FIG. 14. Power spectrum $W(f)$ of the $w(t)$ trajectory for a one-photon dominant process in the time interval $t=(0,100 T)$. The physical parameters used are $\omega_{0}=1.0, \omega=\omega_{0}, k=0.1$, and $\lambda=1.0 \omega_{0}$. 


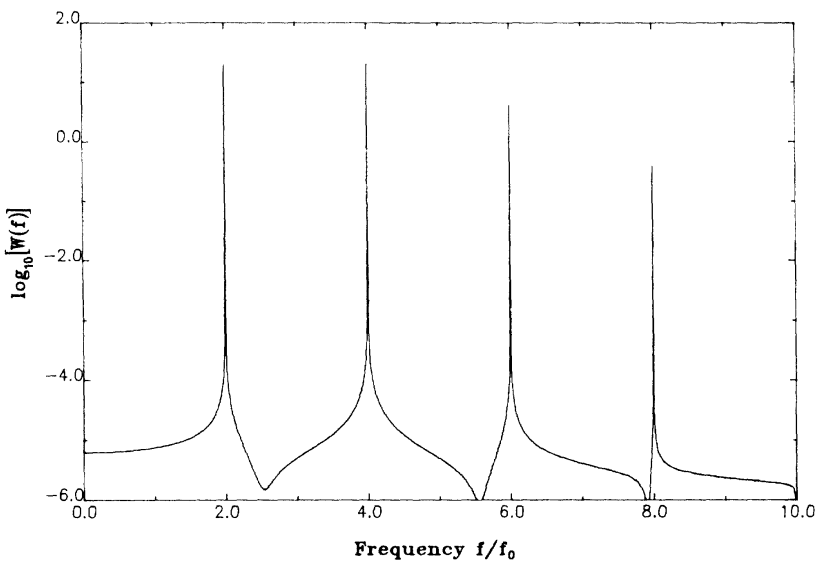

FIG. 15. Same as Fig. 14 except the power spectrum is obtained in the longer time interval $t=(100 T, 200 T)$.

Equation (51) means that, in the rotating frame, the Bloch vector $\mathbf{S}=(u, v, w)$ has a period $\tau^{\prime}=T / 2$. Thus the corresponding fundamental frequency will be

$$
\omega^{\prime}=2 \pi / \tau^{\prime}=2(2 \pi / T)=2 \omega .
$$

This is what we have observed earlier for the fundamental frequency of the limit cycle. Thus we conclude that the limit cycle resulting from the Gisin-like nonlinear Schrödinger equation does possess a dynamical symmetry. The existence of this dynamical symmetry leads to the suppression of the irregular or chaotic behavior in the quantum system.

\section{DISCUSSION AND CONCLUSION}

In this paper we have investigated at length the nonlinear dynamics of dissipative quantum spin systems subject to deterministic nonunitary friction term and periodically driven perturbations. The quantum friction term

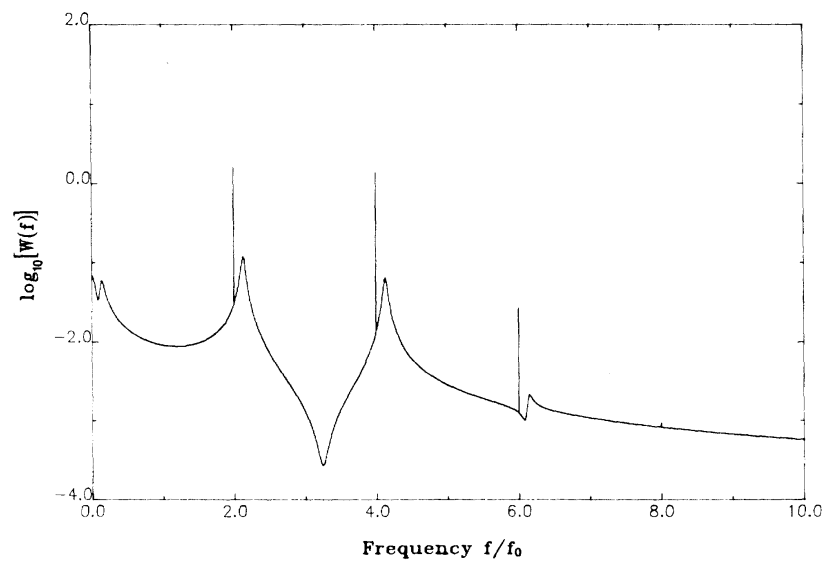

FIG. 16. Power spectrum $W(f)$ of the $w(t)$ trajectory for a three-photon dominant process in the time interval $t=(0,200 T)$. The physical parameters used are $\omega_{0}=1.0$, $\omega=\omega_{0} / 3, k=0.01$, and $\lambda=1.0 \omega_{0}$.

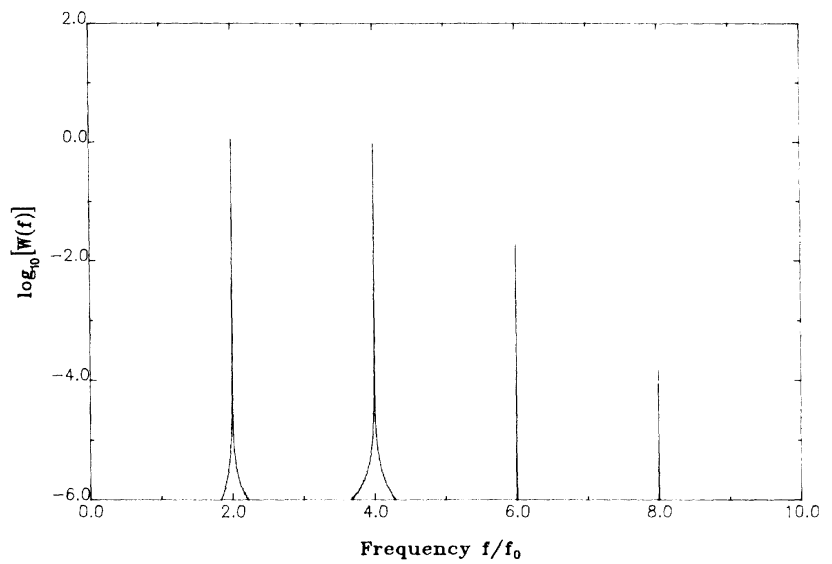

FIG. 17. Same as Fig. 16, except for the longer-time interval $t=(200 T, 400 T)$.

can be considered as the quantum generalization of the classical Landau-Lifshitz frictional resistance. ${ }^{9}$ The presence of this friction term for quantum spin motions leads to (a) the settlement of the system to a limit cycle whose structure depends upon external field frequency, intensity, and order of nonlinear multiphoton transitions, and (b) the confinement of the limit cycle to $\pi / 2<\phi<3 \pi / 2$ (see, for example, Figs. 5-13). This confinement results in the minimization of the interaction energy between the system and the fields. Further we found that the quantum limit cycle exhibits dynamical symmetry and that $\psi(t)$ exhibits a fractal-like evolution pattern in the course of time evolution.

The full consideration of the evolution of dissipative quantum systems should include both a friction term $V_{\mathrm{fr}}$ and a fluctuation term $F$,

$$
\frac{d \psi(t)}{d t}=-i \hat{H}(t) \psi(t)+V_{\mathrm{fr}}+F
$$

The friction term is nonlinear, nonunitary, and deterministic. It forces the system to settle to some "preferred states" (limit cycles) as we have explored in this paper. The fluctuation terms (not considered in this paper) are stochastic and unitary and tend to let the system explore the whole state space. [Spontaneous decay relaxations are also not considered in this paper. They usually can be safely ignored, however, in spin systems. They can be included in the Gisin-like equation (53).] In roomtemperature experiments, the fluctuation effects can mask the nonlinear friction effect. Thus the nonlinear friction effects can best be observed at very low temperatures and for highly polarized samples. An example is given by Redfield's solid-state NMR experiment ${ }^{18}$ performed at high radio-frequency ( $\mathrm{rf}$ ) field intensity. He found the dispersion made $[u(t)]$ of the NMR signal does not saturate at the same level as the absorption $[v(t)]$ but remains roughly constant at high $\mathrm{rf}$ intensities. This cannot be explained by the Bloch's phenomenological theory (in terms of simple $T_{1}$ and $T_{2}$ relaxations) which predicts that the absorption and dispersion should decrease (near resonance) at the same level. A detailed study ${ }^{15}$ of our 
data presented in this paper shows that as the field intensity is increased, $v(t)$ (absorption) can become saturated and decreased while $u(t)$ (dispersion) is not. [Note that $u(t)$ and $v(t)$ are related to the angle $\phi(t)$ defined in Eq. (44).] Thus the nonlinear quantum friction effect offers a possible explanation of Redfield's observations. ${ }^{18}$ Indeed, Gisin $^{6,7}$ has pointed out that the way of introducing friction at a microscopic level [Eq. (3)] leads to a natural unification of Bloch and Redfield phenomenological theories. As emphasized by Abragam ${ }^{19}$ and others, the problem of describing an irreversible dissipative behavior starting from the Schrödinger equation is far from being solved. The proper introduction of effective dissipation in many-body quantum systems is thus a significant problem which has fundamental bearing on the foundations of quantum statistical mechanics. Extension of the Gisinlike theory to many-body atomic, molecular, and optical processes is currently underway.

\section{ACKNOWLEDGMENTS}

This work was supported in part by the U.S. Department of Energy (Division of Chemical Sciences), and for S.I.C. by the John Simon Guggenheim Foundation.
*Also at Department of Physics, University of Kansas, Lawrence, KS 66045.

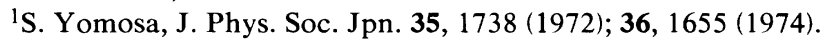

${ }^{2}$ M. D. Newton, J. Chem. Phys. 58, 5833 (1973).

${ }^{3}$ P. R. Surjan and J. Angyan, Phys. Rev. A 28, 45 (1983), and references therein.

${ }^{4}$ See, for example, O. Tapia and G. Johannin, J. Chem. Phys. 75, 3624 (1981); J. Angyan, M. Allavena, M. Picard, A. Potier, and O. Tapia, ibid. 77, 4723 (1982).

5J. G. Kirkwood, J. Chem. Phys. 2, 351 (1934); L. Onsager, J. Am. Chem. Soc. 58, 1486 (1936).

${ }^{6}$ N. Gisin, J. Phys. A 14, 2259 (1981); Physica 111A, 364 (1982); Found. Phys. 13, 643 (1983).

${ }^{7}$ N. Gisin, J. Phys. A 19, 205 (1986).

${ }^{8}$ J. O. Hirschfelder, Adv. Chem. Phys. 73, 1 (1989).

${ }^{9}$ L. D. Landau and E. M. Lifshitz, Z. Phys. 8, 153 (1935); A. Kawabata, Prog. Theor. Phys. 48, 2237 (1972).

${ }^{10}$ J. H. Shirley, Phys. Rev. 138, B979 (1965).
${ }^{11}$ D. R. Dion and J. O. Hirschfelder, Adv. Chem. Phys. 35, 265 (1976).

${ }^{12}$ S. I. Chu, Adv. At. Mol. Phys. 21, 197 (1985); Adv. Chem. Phys. 73, 739 (1989).

${ }^{13}$ K. Aravind and J. O. Hirschfelder, J. Phys. Chem. 88, 4788 (1984), and references therein.

${ }^{14}$ T. S. Ho and S. I. Chu, Phys. Rev. A 31, 659 (1985).

${ }^{15}$ Y. Huang and S. I. Chu (unpublished).

${ }^{16}$ Another example is the quantum suppression of classical chaotic excitation in the kicked-rotator model. See, for example, S. Fishman, D. R. Grempel, and R. E. Prange, Phys. Rev. A 29, 1639 (1984).

${ }^{17}$ See, for example, J. W. Swift and K. Wiesenfeld, Phys. Rev. Lett. 52, 705 (1984).

${ }^{18}$ A. G. Redfield, Phys. Rev. 98, 1787 (1955).

${ }^{19}$ A. Abragam, Principles of Nuclear Magnetism (Oxford University, London, 1983). 